\title{
The Aron-Berner Extension for Polynomials Defined in the Dual of a Banach Space
}

\author{
By \\ Jose G. LlavonA* and Luiza A. Moraes**
}

\begin{abstract}
Let $E=F^{\prime}$ where $F$ is a complex Banach space and let $\pi_{1}: E^{\prime \prime}=E \oplus F^{\perp} \rightarrow E$ be the canonical projection. Let $P\left({ }^{n} E\right)$ be the space of the complex valued continuous $n$-homogeneous polynomials defined in $E$. We characterize the elements $P \in P\left({ }^{n} E\right)$ whose Aron-Berner extension coincides with $P \circ \pi_{1}$. The case of weakly continuous polynomials is considered. Finally we also study the same problem for holomorphic functions of bounded type.
\end{abstract}

\section{Introduction}

Let $E$ be a complex Banach space and let $P\left({ }^{n} E\right)$ denote the space of all continuous $n$-homogeneous polynomials from $E$ into $\mathbb{C}$. The problem of extending every element of $P\left({ }^{n} E\right)$ to a continuous $n$-homogeneous polynomial $\bar{P}$ on the bidual $E^{\prime \prime}$ of $E$ was first studied by Aron and Berner [2] in 1978, who showed that such extensions always exist. The Aron-Berner extension has received most attention since then. Beyond being of interest in its own sight, it is very useful in many branches of complex analysis. Meanwhile it is sometimes hard to work

Communicated by T. Kawai. Received January 7th, 2003.

2000 Mathematics Subject Classification(s): Primary: 46G25; Secondary: 46G20.

Key words: Homogeneous polynomials; Holomorphic functions; Weak star topology.

*Departamento de Análisis Matemático, Facultad de Matemáticas, Universidad Complutense, 28040 Madrid, Spain.

e-mail: JL_Llavona@mat.ucm.es.

Supported in part by Project BFM2000-0609.

** Instituto de Matemática, Universidade Federal do Rio de Janeiro, CP 68530. CEP 21945970 Rio de Janeiro, R. J. Brazil.

e-mail: luiza@im.ufrj.br.

Supported in part by CNPq, Brazil, Research Grant 300016/82-4. 
with. For instance, it was not until 1989 that Davie and Gamelin [8] showed that the norm of the Aron-Berner extension $\bar{P}$ of $P \in P\left({ }^{n} E\right)$ to $E^{\prime \prime}$ coincides with the norm of $P$. In 1990 Zalduendo [14] gave a characterization of this extension in terms of continuity properties of the first order differentials.

If $E$ is a dual Banach space, it is known that $E$ is complemented in $E^{\prime \prime}$ and clearly the projection $\pi_{1}: E^{\prime \prime} \rightarrow E$ provides a norm-preserving extension of every element of $P\left({ }^{n} E\right)$ to $E^{\prime \prime}$. Our main goal in this paper is to determine necessary and sufficient conditions for the equality $\bar{P}=P \circ \pi_{1}$ be true. As a consequence we solve some related problems. For instance, in a recent paper Aron, Boyd and Choi [3] investigate the unicity of the norm-preserving extensions of elements of $P\left({ }^{n} E\right)$ to $E^{\prime \prime}$. We will get here that if $E$ is a nonreflexive dual Banach space, then for every $n>1$ there exists always an element $P \in P\left({ }^{n} E\right)$ such that $\bar{P} \neq P \circ \pi_{1}$ and so the norm-preserving extension is not always unique in such spaces.

Our notation is as follows. Throughout, $E$ and $G$ will be complex Banach spaces. For $n \in \mathbb{N}, L\left({ }^{n} E, G\right)$ (resp. $L_{s}\left({ }^{n} E, G\right)$ ) denotes the space of all continuous (resp. continuous symmetric) $n$-linear mappings $A: E^{n} \rightarrow G$. For each $\left(x_{1}, \ldots, x_{k}\right) \in E^{k}$ and each $\alpha=\left(\alpha_{1}, \ldots, \alpha_{k}\right) \in \mathbb{N}^{k}$ with $\alpha_{1}+\cdots+\alpha_{k}=n$ we will write $A\left(x_{1}\right)^{\alpha_{1}} \ldots\left(x_{k}\right)^{\alpha_{k}}$ for $A\left(x_{1},{ }^{\alpha_{1}}, x_{1}, \ldots, x_{k}, \alpha_{k}, x_{k}\right)$. For $n \in \mathbb{N}$, let $P\left({ }^{n} E, G\right)$ be the space of all $P: E \rightarrow G$ such that $P(x)=A(x)^{n} \forall x \in E$ for some $A \in L\left({ }^{n} E, G\right)$. The elements of this space are called the continuous $n$-homogeneous polynomials. The space $P\left({ }^{n} E, G\right)$ is a Banach space under the norm $\|P\|=\sup \{\|P(x)\|: x \in E,\|x\| \leq 1\}$. By definition $L\left({ }^{0} E, G\right)=$ $G=P\left({ }^{0} E, G\right)$. Given $P \in P\left({ }^{n} E, G\right)$, it is straightforward that we may take the associated $n$-linear mapping $A$ to be symmetric; in fact $P\left({ }^{n} E, G\right)$ and $L_{s}\left({ }^{n} E, G\right)$ are isomorphic as Banach spaces. The unique associated symmetric $n$-linear mapping $\breve{P}$ is given by the Polarization Formula. It is well known that $\|P\| \leq\|\breve{P}\| \leq \frac{n^{n}}{n !}\|P\|$.

Given any $P \in P\left({ }^{n} E, G\right)$, let $T_{P}: E \rightarrow L_{s}\left({ }^{n-1} E, G\right)$ be the linear bounded operator given by $T_{P}(x)\left(x_{1}, \ldots, x_{n-1}\right):=\breve{P}\left(x, x_{1}, \ldots, x_{n-1}\right)$ and let $d P \in$ $P\left({ }^{n-1} E, L(E, G)\right)$ its derivative. By the symmetry of $\breve{P}$ it is obvious that $d P(x)(y)=n \breve{P}(x)^{n-1}(y)=n T_{P}(y)(x)^{n-1}$. Its associated operator $T_{d P}: E \rightarrow$ $L_{s}\left({ }^{n-2} E, L(E, G)\right)$ is given by $T_{d P}(x)\left(x_{1}, \ldots, x_{n-2}\right)=n \breve{P}\left(x, x_{1}, \ldots, x_{n-2}, \bullet\right)$.

Throughout, if the range space is not specifically identified, then it will be assumed that the range is $\mathbb{C}$; thus, for example, $L\left({ }^{n} E\right)$ denotes $L\left({ }^{n} E, \mathbb{C}\right), P\left({ }^{n} E\right)$ denotes $P\left({ }^{n} E, \mathbb{C}\right)$, etc.

If $E=F^{\prime}$ for some Banach space $F$, we have $E^{\prime \prime}=E \oplus F^{\perp}$. In this case the weak topology associated to the dual pair $(E, F)$ will be denoted 
by $w^{*}$. Let $\pi_{1}: E^{\prime \prime} \rightarrow E$ be the canonical projection. As usual we denote by $\widehat{\bigotimes}_{n, \pi} E$ (resp. $\widehat{\bigotimes}_{n, \pi, s} E$ ) the completion of the $n$-fold (resp. symmetric $n$-fold) tensor product of $E$, with the projective norm. We remark that the projective norm in $\widehat{\otimes}_{n, \pi, s} E$ is defined by $\pi_{s}(u)=\inf \left\{\sum_{k=1}^{\infty}\left\|x_{k}\right\|^{m}\right.$ : $\left.u=\sum_{k=1}^{\infty} \varepsilon_{k} x_{k} \otimes \cdots \otimes x_{k} ; \varepsilon_{k}= \pm 1\right\}$, where the infimum is taken over all such representations $u=\sum_{k=1}^{\infty} \varepsilon_{k} x_{k} \otimes \cdots \otimes x_{k} \in \widehat{\otimes}_{n, \pi, s} E$. This norm is different of the restriction to $\widehat{\otimes}_{n, \pi, s} E$ of the usual projective norm defined in $\widehat{\otimes}_{n, \pi} E$. We refer to [9] or [11] for details. It is known that $L\left({ }^{n-1} E\right)=\left(\widehat{\bigotimes}_{n-1, \pi} E\right)^{\prime}$ and $L_{s}\left({ }^{n-1} E\right)=\left(\widehat{\bigotimes}_{n-1, \pi, s} E\right)^{\prime}$. The weak topology on $L\left({ }^{n-1} E\right)$ associated to the dual pair $\left(L\left({ }^{n-1} E\right), \widehat{\bigotimes}_{n-1, \pi} E\right)$ will be also denoted by $w^{*}$. Given any $P \in P\left({ }^{n} E\right), \quad \bar{P}$ will always denote the Aron-Berner extension of $P$ to $E^{\prime \prime}$ (cf. [2]).

For basic results and unclear notation we refer to [13].

\section{$\S 1$ The Extension of Polynomials}

Theorem 1.1. Suppose that $E=F^{\prime}$ for some Banach space $F$. Then for $P \in P\left({ }^{n} E\right)$ the following conditions are equivalent:

(1) $T_{P}$ is $w^{*}-w^{*}$-continuous.

(2) $d P(x)$ is $w^{*}$-continuous $\forall x \in E$.

(3) $d P(x) \in F \quad \forall x \in E$.

(4) $\bar{P}=P \circ \pi_{1}$.

Proof. $\quad(1) \Rightarrow(2)$. Let $\left(x_{\alpha}\right) \subset E$ such that $\left(x_{\alpha}\right) \stackrel{w^{*}}{\rightarrow} z \in E$. By (1) we have that $(x \otimes \cdots \otimes x)\left(T_{P}\left(x_{\alpha}\right)\right) \rightarrow(x \otimes \cdots \otimes x)\left(T_{P}(z)\right) \forall x \otimes \cdots \otimes x \in \widehat{\bigotimes}_{n-1, \pi} E$ and consequently $d P(x)\left(x_{\alpha}\right)=n T_{P}\left(x_{\alpha}\right)(x, \cdots, x)=(x \otimes \cdots \otimes x)\left(n T_{P}\left(x_{\alpha}\right)\right) \rightarrow$ $(x \otimes \cdots \otimes x)\left(n T_{P}(z)\right)=d P(x)(z)$.

$(2) \Rightarrow(3)$ is clear.

$(3) \Rightarrow(4)$. By [14], all we have to show is

(i) $\forall x \in E, \quad d\left(P \circ \pi_{1}\right)(x)$ is $w^{*}$-continuous.

and

(ii) $\forall z \in E^{\prime \prime}$ and $\forall\left(x_{\alpha}\right) \subset E$ such that $\left(x_{\alpha}\right) w^{*} \rightarrow z$ we have $d(P \circ$ $\left.\pi_{1}\right)(z)\left(x_{\alpha}\right) \rightarrow d\left(P \circ \pi_{1}\right)(z)(z)$. Take $\left(z_{\alpha}\right) \subset E^{\prime \prime}$ such that $\left(z_{\alpha}\right) \stackrel{w^{*}}{\rightarrow} z \in E^{\prime \prime}$. As $E^{\prime \prime}=E \oplus F^{\perp}$ we can write $z=\pi_{1}(z)+z_{2}$ where $z_{2} \in F^{\perp}$ and $z_{\alpha}=\pi_{1}\left(z_{\alpha}\right)+z_{\alpha}^{\prime}$ where $z_{\alpha}^{\prime} \in F^{\perp} \forall \alpha$. For every $x \in E, \quad d\left(P \circ \pi_{1}\right)(x)\left(z_{\alpha}\right)=d P(x)\left(\pi_{1}\left(z_{\alpha}\right)\right)=$ 
$\pi_{1}\left(z_{\alpha}\right)(d P(x))=z_{\alpha}(d P(x))$ since $z_{\alpha}^{\prime} \in F^{\perp}$ and $d P(x) \in F$. But $\left(z_{\alpha}\right) \stackrel{w^{*}}{\rightarrow} z$ and $d P(x) \in E^{\prime}$ implies $z_{\alpha}(d P(x)) \rightarrow z(d P(x))=\left(\pi_{1}(z)+z_{2}\right)(d P(x))=$ $d P(x)\left(\pi_{1}(z)\right)=d\left(P \circ \pi_{1}\right)(z)$ and this gives $(\mathrm{i})$.

Now fix $z \in E^{\prime \prime}$ arbitrary and take $\left(x_{\alpha}\right) \subset E$ such that $\left(x_{\alpha}\right) \stackrel{w^{*}}{\rightarrow} z$. Let $z=\pi_{1}(z)+z_{2} \quad$ where $z_{2} \in F^{\perp}$. Then we have $d\left(P \circ \pi_{1}\right)(z)\left(x_{\alpha}\right)=$ $d P\left(\pi_{1}(z)\right)\left(\pi_{1}\left(x_{\alpha}\right)\right)=d P\left(\pi_{1}(z)\right)\left(x_{\alpha}\right) \rightarrow z\left(d P\left(\pi_{1}(z)\right)\right)=\left(\pi_{1}(z)+z_{2}\right)$ $\left(d P\left(\pi_{1}(z)\right)\right)=d P\left(\pi_{1}(z)\right)\left(\pi_{1}(z)\right)=d\left(P \circ \pi_{1}\right)(z)(z)$ and this completes the proof of (ii).

$(4) \Rightarrow(3)$. Take $x \in E$ and $z_{2} \in F^{\perp}$ arbitrary and consider $z=x+z_{2} \in E^{\prime \prime}$. By [14] we have that $d\left(P \circ \pi_{1}\right)(z)\left(x_{\alpha}\right) \rightarrow d\left(P \circ \pi_{1}\right)(z)(z)$ for every $\left(x_{\alpha}\right) \subset E$ such that $\left(x_{\alpha}\right) \stackrel{w^{*}}{\rightarrow} z$. Since $d P(x) \in E^{\prime}$ we have $d\left(P \circ \pi_{1}\right)(z)\left(x_{\alpha}\right)=d P(x)\left(x_{\alpha}\right) \rightarrow$ $z(d P(x))=\left(\pi_{1}(z)+z_{2}\right)(d P(x))=d P(x) \circ \pi_{1}(z)+z_{2}(d P(x))$ and the unicity of the limit gives $z_{2}(d P(x))=0$. As $z_{2} \in F^{\perp}$ was arbitrary, by using the Hahn-Banach theorem we get $d P(x) \in F$.

$(2) \Rightarrow(1)$ Let $\left(x_{\alpha}\right) \subset E$ such that $\left(x_{\alpha}\right) \stackrel{w^{*}}{\rightarrow} z \in E$. By (2) we have $(x \otimes$ $\cdots \otimes x)\left(T_{P}\left(x_{\alpha}\right)\right)=T_{P}\left(x_{\alpha}\right)(x, \ldots, x)=\breve{P}\left(x, \ldots, x, x_{\alpha}\right)=\frac{1}{n} d P(x)\left(x_{\alpha}\right) \longrightarrow$ $\frac{1}{n} d P(x)(z)=T_{P}(z)(x, \ldots, x)=(x \otimes \cdots \otimes x)\left(T_{P}(z)\right) \quad \forall x \in E$. Now, by using the polarization formula we have that $\left(x_{1} \otimes \cdots \otimes x_{n-1}\right)\left(T_{P}\left(x_{\alpha}\right)\right) \rightarrow$ $\left(x_{1} \otimes \cdots \otimes x_{n-1}\right)\left(T_{P}(z)\right) \quad \forall x_{1} \otimes \cdots \otimes x_{n-1} \in \widehat{\bigotimes}_{n-1, \pi} E$ and this implies (1).

Example 1.1. Suppose that $E=F^{\prime}$ for some nonreflexive Banach space $F$. Consider $P: E \rightarrow \mathbb{K}$ defined by $P(x):=\varphi^{n}(x) \quad \forall x \in E$ where $\varphi \in E^{\prime}$. It is clear that $\bar{P}=P \circ \pi_{1} \quad$ if $\quad \varphi \in F \quad$ and $\quad \bar{P} \neq P \circ \pi_{1} \quad$ if $\varphi \notin F$.

Example 1.2. For $E=l_{1}=c_{0}^{\prime}$ every diagonal $n$-homogeneous polynomial $P: l_{1} \rightarrow \mathbb{K}$ satisfies $\bar{P}=P \circ \pi_{1}$. Indeed: We recall that a diagonal $n$-homogeneous polynomial $P$ is defined by $P(x)=\sum_{j=1}^{\infty} a_{j} x_{j}^{n}$ where $\left(a_{j}\right) \in l_{\infty}$. Now, $T_{P}: l_{1} \rightarrow\left(\widehat{\bigotimes}_{n-1, \pi} l_{1}\right)^{\prime}$ is defined by $T_{P}(x)\left(z^{1} \otimes \cdots \otimes z^{n-1}\right)=$ $\sum_{j=1}^{\infty} a_{j} x_{j} z_{j}^{1} \ldots z_{j}^{n-1}$ where $x=\left(x_{j}\right)_{j=1}^{\infty}$ and $z^{i}=\left(z_{j}^{i}\right)_{j=1}^{\infty} \forall 1 \leq i \leq n-1$ and it is clear that $T_{P}=B^{*}$ where $B: \widehat{\bigotimes}_{n-1, \pi} l_{1} \rightarrow c_{0}$ is defined by $B\left(z^{1} \otimes \cdots \otimes z^{n-1}\right)=$ $\left(a_{i} z_{i}^{1} \ldots z_{i}^{n-1}\right)_{i=1}^{\infty}$. Consequently $T_{P}$ is $w^{*}-w^{*}$ continuous.

Example 1.3. Suppose that $k \in \mathbb{N}$ and consider the sequence $\left(A_{j}\right)_{j=1}^{\infty}$, $A_{j} \in L_{s}\left({ }^{k-1} l_{1}\right)$, satisfying: 
(1.3.1) $A_{j}$ is symmetric in the sense that $A_{i_{1}}\left(e_{i_{2}}, \ldots, e_{i_{k}}\right)$ is invariant under permutation of the indices $i_{1}, \ldots, i_{k}$.

(1.3.2) $\left(A_{j}\right)_{j=1}^{\infty}$ is equivalent to the unit vector basis of $l_{1}$.

(1.3.3) For all indices $i_{1}, \ldots, i_{k}, A_{i_{1}}\left(e_{i_{2}}, \ldots, e_{i_{k}}\right) \in\{-1,1\}$.

We refer to [12] for the proof of the existence of such a sequence.

If $P \in P\left({ }^{k} l_{1}\right)$ is given by

$$
P(\alpha):=\sum_{i_{1}, \ldots, i_{k}=1}^{\infty} \alpha_{i_{1}} \ldots \alpha_{i_{k}} A_{i_{1}}\left(e_{i_{2}}, \ldots, e_{i_{k}}\right) ; \text { for } \alpha=\left(\alpha_{j}\right)_{j=1}^{\infty} \in l_{1}
$$

it follows that $\bar{P} \neq P \circ \pi_{1}$. Indeed, it is enough to observe that for all $i \in \mathbb{N}, \quad A_{j}(\overbrace{e_{i}, \ldots, e_{i}}^{k-1}) \in\{-1,1\} \quad$ and therefore $T_{P}: l_{1} \rightarrow\left(\widehat{\bigotimes}_{k-1, \pi} l_{1}\right)^{\prime} \quad$ is given by $T\left(e_{j}\right)=A_{j}$ and clearly is not $w^{*}-w^{*}$ continuous.

Aron and Prolla [6] showed that the finite type polynomials on a Banach space are exactly the weakly continuous polynomials. The proof of next lemma will be omitted as it is very similar to the proof given by Aron and Prolla for their result. This lemma will be used in the proof of Theorem 1.3.

Lemma 1.2. $\quad$ Suppose that $E=F^{\prime}$ for some Banach space $F$ and let $P \in P\left({ }^{n} E\right)$. Then $P$ is $w^{*}$-continuous on $E$ if and only if there exists $\phi_{1}, \ldots$, $\phi_{M} \in F$ such that $P=\sum_{i=0}^{M} \phi_{i}^{n}$.

Theorem 1.3. $\quad$ Suppose that $E=F^{\prime}$ for some Banach space $F$ and $P$ is a weakly continuous $n$-homogeneous polynomial from $E$ into $\mathbb{C}$. Then $P$ is $w^{*}$-continuous if and only if $d P(x) \in F \quad \forall x \in E$.

Proof. We are going to use the induction.

The statement is clearly true for $n=1$. Assume that for every $k=$ $1,2, \ldots, n-1$ it is true. Since $P$ is weakly continuous, there are functionals $\phi_{1}, \ldots, \phi_{N} \in E^{\prime}$ such that $P=\sum_{i=1}^{N} \phi_{i}^{n}$. For all $x \in E, d P(x)=\sum_{i=1}^{N}$ $n \phi_{i}^{n-1}(x) \phi_{i}$. Therefore the condition $d P(x) \in F \quad \forall x \in E$ means that there exists a linearly independent subset $\left\{\psi_{1}, \ldots, \psi_{M}\right\}$ of $F$ such that $d P(E) \subset$ $\operatorname{span}\left\{\psi_{1}, \ldots, \psi_{M}\right\}$. Let $x_{1}, \ldots, x_{M} \in E$ such that $\psi_{i}\left(x_{j}\right)=\delta_{i j},(1 \leq i, j \leq$ $M)$. For each $x \in E$ we have $d P(x)=\sum_{j=1}^{M} \lambda_{j}(x) \psi_{j}$ where for all $j=$ $1, \ldots, M$ we have $\lambda_{j}(x)=d P(x)\left(x_{j}\right)=n T_{P}\left(x_{j}\right)(x)^{n-1}$ for all $x \in E$. It is clear that each $\lambda_{j} \in P\left({ }^{n-1} E\right)$ and $\lambda_{j}$ is $w$-continuous. Now, as $d \lambda_{j}(x)=$ $d^{2} P(x)\left(x_{j}\right) \in F \quad \forall x \in E$, by the hypothesis of induction it follows that $\lambda_{j}$ is $w^{*}$ continuous and this is true for all $j=1, \ldots, M$. Finally, $P(x)=T_{P}(x)(x)^{n-1}=$ 
$\frac{1}{n} d P(x)(x)=\frac{1}{n} \sum_{j=0}^{M} \lambda_{j}(x) \psi_{j}(x)$ is $w^{*}$-continuous since $\psi_{j} \in F$ and $\lambda_{j}$ is $w^{*}$ continuous $\forall j=1, \ldots, M$. So the proof is complete. The proof of the converse follows trivially as a consequence of Lemma 1.2.

Corollary 1.4. $\quad$ Suppose that $E=F^{\prime}$ for some Banach space $F$. Then for every weakly continuous n-homogeneous polynomial $P$ from $E$ into $\mathbb{C}$ we have that $\bar{P}=P \circ \pi_{1}$ if and only if $P$ is $w^{*}$-continuous.

Remark 1.1. In connection with Theorem 1.3, it is reasonable to ask if every sequentially weakly continuous $n$-homogeneous polynomials $P$ from $E$ into $\mathbb{C}$ such that $d P(x) \in F \quad \forall x \in E$ is sequentially $w^{*}$-continuous. The answer is negative. Indeed: Recall that every continuous mapping defined in $l_{1}$ is sequentially weakly continuous. Now take $P: l_{1} \rightarrow \mathbb{C}$ defined by $P(x)=\sum_{n=1}^{\infty} x_{n}^{2} \quad \forall x=\left(x_{n}\right)_{n=1}^{\infty} \in l_{1}$. By Example 1.2, $d P(x) \in F \quad \forall x \in l_{1}$, but $P$ is not $w^{*}$-sequentially continuous as $P\left(e_{n}\right)=1 \forall n \in \mathbb{N}$.

We denote by $P_{w u}\left({ }^{n} E, G\right)$ the subspace of those elements of $P\left({ }^{n} E, G\right)$ whose restrictions to the bounded subsets of $E$ are (uniformly) weakly continuous. It is well known that every such polynomial is compact and, in case $n=1, T \in P_{w u}\left({ }^{1} E, G\right)$ if and only if $T: E \rightarrow G$ is a compact linear operator (see [6] Lemma 2.2 and Proposition 2.5). For every $n \in \mathbb{N}$ it is true that $P \in P_{w u}\left({ }^{n} E\right)$ if and only if the associated operator $T_{P}$ is compact (see [4] Theorem 2.9). We will use the fact that $P \in P_{w u}\left({ }^{n} E\right)$ if and only if the associated operator $T_{P}$ is compact (see [4], Theorem 2.9).

Theorem 1.5. Suppose that $E=F^{\prime}$ for some Banach space F. If $P \in$ $P\left({ }^{n} E\right)$ is weakly continuous on bounded sets and $d P(x) \in F \forall x \in E$ then $P$ is $w^{*}$-continuous on the bounded subsets of $E$.

Proof. If $P \in P\left({ }^{n} E\right)$, its derivative $d P \in P\left({ }^{n-1} E, E^{\prime}\right)$ and $d P(x)(y)=$ $n T_{P}(y)(x)^{n-1}=n T_{P}(x)(x)^{n-2}(y)$. The condition $d P(x) \in F \forall x \in E$, means that the continuous linear operator $\widetilde{d P}: \widehat{\bigotimes}_{n-1, \pi, s} E \rightarrow F$, $\widetilde{d P}(x \otimes \cdots \otimes x)=$ $d P(x)$ is well defined. We consider now the adjoint operator

$$
\widetilde{d P}^{*}: F^{\prime} \rightarrow\left(\widehat{\bigotimes}_{n-1, \pi, s} E\right)^{\prime}=L_{s}\left({ }^{n-1} E\right)=P\left({ }^{n-1} E\right)
$$

It is clear that $\widetilde{d P}^{*}(x)(y \otimes \cdots \otimes y)=d P(y)(x), \quad x, y \in E$. Also $d P$ is compact if and only if $\widetilde{d P}^{*}$ is compact (see [7]). 
If $\left(x_{\alpha}\right) \subset E$ is a bounded net such that $\left(x_{\alpha}\right) \rightarrow x$ weakly, and $y \in E$ we have $\frac{1}{n}\left|d P\left(x_{\alpha}\right)(y)-d P(x)(y)\right|=\left|T_{P}\left(x_{\alpha}\right)\left(x_{\alpha}\right)^{n-2}(y)-T_{P}(x)(x)^{n-2}(y)\right|=$ $\mid T_{P}\left(x_{\alpha}-x\right)\left(x_{\alpha}\right)^{n-2}(y)+T_{P}\left(x_{\alpha}-x\right)\left(x_{\alpha}\right)^{n-3}(x)(y)+\cdots+T_{P}\left(x_{\alpha}-x\right)$ $\left(x_{\alpha}\right)(x)^{n-3}(y)+T_{P}\left(x_{\alpha}-x\right)(x)^{n-2}(y) \mid$.

The compactness of $T_{P}$ means that $T_{P}$ is weakly- $\|\cdot\|$ continuous on bounded sets, and therefore

$$
\left\|d P\left(x_{\alpha}\right)-d P(x)\right\|=\sup _{y \in E,\|y\| \leq 1}\left\|d P\left(x_{\alpha}\right)(y)-d P(x)(y)\right\| \stackrel{\alpha}{\longrightarrow} 0
$$

and $d P$ is compact. The compactness of $d P$ means that $\widetilde{d P}$ is compact since every Banach space has $(B B)_{\infty}\left(\right.$ see $[10]$ p. 32) and therefore $\widetilde{d P}^{*}$ is $w^{*}$ $\|\cdot\|$ continuous on $w^{*}$-compact sets in $F^{\prime}=E$. We remark that for $x \in E$,

$$
\begin{aligned}
\left\|\widetilde{d P}^{*}(x)\right\| & =\sup _{y \in E,\|y\| \leq 1}|d P(y)(x)| \\
& =n \sup _{y \in E,\|y\| \leq 1}\left|T_{P}(x)(y)^{n-1}\right|=n\left\|T_{P}(x)\right\| .
\end{aligned}
$$

If $\left(x_{\alpha}\right) \subset E$ is a bounded net such that $\left(x_{\alpha}\right) \stackrel{w^{*}}{\rightarrow} x$, we have that $\widetilde{d P}^{*}\left(x_{\alpha}\right) \rightarrow$ $\widetilde{d P}^{*}(x)$ in norm and therefore

$$
\begin{aligned}
\left|P\left(x_{\alpha}\right)-P(x)\right|= & \left|T_{P}\left(x_{\alpha}\right)\left(x_{\alpha}\right)^{n-1}-T_{P}(x)(x)^{n-1}\right| \\
= & \mid T_{P}\left(x_{\alpha}-x\right)\left(x_{\alpha}\right)^{n-1}+T_{P}\left(x_{\alpha}-x\right)(x)\left(x_{\alpha}\right)^{n-2} \\
& +\cdots+T_{P}\left(x_{\alpha}-x\right)(x)^{n-2}\left(x_{\alpha}\right)+T_{P}\left(x_{\alpha}-x\right)(x)^{n-1} \mid \stackrel{\alpha}{\longrightarrow} 0,
\end{aligned}
$$

because

$$
\left\|T_{P}\left(x_{\alpha}-x\right)\right\|=\left\|T_{P}\left(x_{\alpha}\right)-T_{P}(x)\right\| \leq \frac{1}{n}\left\|\widetilde{d P}^{*}\left(x_{\alpha}\right)-\widetilde{d P}^{*}(x)\right\| \stackrel{\alpha}{\longrightarrow} 0,
$$

and the proof is complete.

Corollary 1.6. Suppose that $E=F^{\prime}$ for some Banach space F. For $P \in P_{w b}\left({ }^{n} E\right)$ we have that $P$ is $w^{*}$-continuous on the bounded subsets of $E$ whenever $\bar{P}=P \circ \pi_{1}$.

Let $P_{w^{*} b}\left({ }^{n} E\right)$ be the space of the elements of $P\left({ }^{n} E\right)$ that are $w^{*}$-continuous on the bounded subsets of $E$.

Definition 1.1. A Banach space $E$ is said to have the Mazur's property if $w^{*}$-sequentially continuous functionals on $E^{\prime}$ are actually $w^{*}$-continuous, i.e. belong to $E$. 
Every separable Banach space enjoy Mazur's property while $l_{\infty}$ doesn't have it.

Proposition 1.7. $\quad$ Suppose that $E=F^{\prime}$ for some Banach space $F$. If $F$ has the Mazur's property, for every $P \in P_{w^{*} b}\left({ }^{n} E\right)$ we have that $d P(x)$ is $w^{*}$-continuous $\forall x \in E$ and, consequently, $\bar{P}=P \circ \pi_{1}$.

Proof. Since $F$ has the Mazur's property, it is enough to show that $d P(x)$ is $w^{*}$-sequentially continuous in $E$. Let $\left(y_{k}\right) \stackrel{w^{*}}{\rightarrow} y$ in $E$ and let $\epsilon>0$. If there exists $k_{0} \in \mathbb{N}$ such that

$$
\sup _{|\lambda|=1}\left|P\left(x+\lambda y_{k}\right)-P(x+\lambda y)\right|<\epsilon \quad \forall k \geq k_{0},
$$

the thesis follows by noting that

$$
\left|d P(x)\left(y_{k}\right)-d P(x)(y)\right|=\frac{1}{2 \pi}\left|\int_{|\lambda|=1} \frac{P\left(x+\lambda y_{k}\right)-P(x+\lambda y)}{\lambda^{2}} d \lambda\right| .
$$

Suppose that $(*)$ is not true for every $k_{0} \in \mathbb{N}$. In this case, for infinitely many $k \in \mathbb{N}$ there exist $\left|\lambda_{k}\right|=1$ such that $\left|P\left(x+\lambda_{k} y_{k}\right)-P\left(x+\lambda_{k} y\right)\right| \geq \epsilon$. But $\left(\lambda_{k}\right)$ has a subsequence $\left(\lambda_{k_{i}}\right)$ converging to some $\lambda$ satisfying $|\lambda|=1$ and then, since $\left(x+\lambda_{k_{i}} y_{k_{i}}\right) \stackrel{w^{*}}{\rightarrow} x+\lambda y$, and $P$ is $w^{*}$-continuous on bounded subsets of $E$ we have $\left|P\left(x+\lambda_{k_{i}} y_{k_{i}}\right)-P\left(x+\lambda_{k_{i}} y\right)\right| \leq\left|P\left(x+\lambda_{k_{i}} y_{k_{i}}\right)-P(x+\lambda y)\right|+$ $\left|P(x+\lambda y)-P\left(x+\lambda_{k_{i}} y\right)\right| \rightarrow 0$, a contradiction.

Proposition 1.8. Suppose that $E=F^{\prime}$ for some Banach space $F$. Then for $P \in P\left({ }^{n} E\right)$ the following conditions are equivalent:

(1) $\bar{P}=P \circ \pi_{1}$.

(2) $\bar{P}$ factors throughout $F^{\perp}$.

Proof. $\quad(1) \Rightarrow(2)$. Take any $z \in E^{\prime \prime}$. If $\bar{P}=P \circ \pi_{1}, \forall w \in F^{\perp}$ we have $d \bar{P}(z)(w)=d P\left(\pi_{1}(z)\right)(w)=d P\left(\pi_{1}(z)\right)(0)=0$. So $d \bar{P}(z) \in\left(F^{\perp}\right)^{\perp} \forall z \in E^{\prime \prime}$ and $\bar{P}$ factors throughout $F^{\perp}$ by [5], Theorem 2.2 .

$(2) \Rightarrow(1)$. We first remark that $\bar{P}$ factors throughout $F^{\perp}$ means that $\bar{P}$ factors throughout $j\left(F^{\perp}\right)$ where $j: E \rightarrow E^{\prime \prime}$ is the canonical immersion.

We are going to show that $d P(x) \in F \forall x \in E$ and then infer (1) by Theorem 1.1. It is enough to show that $\forall x \in E$ we have $d P(x)(y)=0 \quad \forall y \in F^{\perp}$. By [5], Theorem 2.2 we have that $d \bar{P}(z) \in j\left(F^{\perp}\right)^{\perp} \forall z \in E^{\prime \prime}$. For each $x \in E$ and $y \in F^{\perp} \subset E$ we have $d P(x)(y)=\breve{P}(x, \ldots, x, y)=\bar{P}(j(x))(j(y))=0$ as $j(y) \in j\left(F^{\perp}\right)$. So, $d P(x) \in F \quad \forall x \in E$. 


\section{$\S 2 . \quad$ The Holomorphic Case}

Let $H_{b}(E)$ be the space of the holomorphic mappings that are bounded in the bounded subset of $E$. We denote by $H_{w u}(E)$ (respectively $H_{w^{*} u}(E)$ ) the space of the holomorphic functions from $E$ into $\mathbb{C}$ that are uniformly $w$ continuous (respectively uniformly $w^{*}$-continuous) on the bounded subsets of $E$. Given $f=\sum_{n=0}^{\infty} P_{n} \in H_{b}(E)$, the Aron-Berner extension $\bar{f}$ from $f$ to $E^{\prime \prime}$ is, by definition, the function $\bar{f}:=\sum_{n=0}^{\infty} \bar{P}_{n} \in H_{b}\left(E^{\prime \prime}\right)$.

Theorem 2.1. $\quad$ Suppose that $E=F^{\prime}$ for some Banach space $F$. Then for $f \in H_{b}(E)$ the following conditions are equivalent:

(1) $\bar{f}=f \circ \pi_{1}$

(2) $\quad d f(x) \in F \quad \forall x \in E$.

(3) $d f(x)$ is $w^{*}$-continuous $\forall x \in E$.

Proof. $\quad(1) \Rightarrow(2) . \quad f=\sum_{n=0}^{\infty} P_{n} \in H_{b}(E)$. Let $B \subset E^{\prime \prime}$ be a bounded set. It is clear that $\pi_{1}(B) \subset E$ is bounded and since $f \in H_{b}(E, F)$ we have $\left\|f-\sum_{n=0}^{k} P_{n}\right\|_{\pi_{1}(B)} \rightarrow 0$. So, $\left\|f \circ \pi_{1}-\sum_{n=0}^{k} P_{n} \circ \pi_{1}\right\|_{B} \rightarrow 0 \quad \forall B \subset B^{\prime \prime}$ bounded and consequently $f \circ \pi_{1}=\sum_{n=0}^{\infty} P_{n} \circ \pi_{1} \in H_{b}\left(E^{\prime \prime}\right)$.

But $\bar{f}=\sum_{n=0}^{\infty} \bar{P}_{n}$ and by the unicity of the Taylor representation, $\bar{f}=$ $f \circ \pi_{1}$ if and only if $\bar{P}_{n}=P_{n} \circ \pi_{1}$ for every $n \in \mathbb{N}$. By Theorem 1.1 we have $d P_{n}(x) \in F \quad \forall x \in E \quad \forall n \in \mathbb{N}$ and consequently $d f(x) \in F \quad \forall x \in E$.

$(2) \Rightarrow(1)$. The proof is similar to the proof of $(3) \Rightarrow(4)$ in Theorem 1.1.

$(2) \Leftrightarrow(3)$ is clear.

Proposition 2.2. Suppose that $E=F^{\prime}$ for some Banach space $F$. If $f=\sum_{n=0}^{\infty} P_{n} \in H_{w u}(E)$ and $d f(x) \in F \quad \forall x \in E$ then $f \in H_{w^{*} u}(E)$.

Proof. Let $f=\sum_{n=0}^{\infty} P_{n} \in H_{w u}(E) \subset H_{b}(E)$. Combining Proposition 1.5 (b) of [1] and Theorem 2.1 we obtain that $P_{n} \in P_{w^{*} u}\left({ }^{n} E\right) \forall n \in \mathbb{N}$ and the proposition follows immediately.

Proposition 2.3. $\quad$ Suppose that $E=F^{\prime}$ for some Banach space $F$. If $F$ has the Mazur's property, for every $f \in H_{w^{*} u}(E)$ we have that $d f(x)$ is $w^{*}$-continuous $\forall x \in E$ and, consequently, $\bar{f}=f \circ \pi_{1}$.

Proof. The same of Proposition 1.7. 
Proposition 2.4. $\quad$ Suppose that $E=F^{\prime}$ for some Banach space $F$. Then for $f=\sum_{n=0}^{\infty} P_{n} \in H_{b}(E)$ the following conditions are equivalent:

(1) $\bar{f}=f \circ \pi_{1}$

(2) $\bar{f}$ factors throughout $F^{\perp}$.

Proof. The proof is similar to the proof of Proposition 1.7. Use [5], Theorem 2.3 instead of [5], Theorem 2.2.

\section{Acknowledgement}

Part of this paper was written while the first author was visiting the Universidad Complutense de Madrid. She wishes to thank the Department of Mathematics at that University for their hospitality.

\section{References}

[1] Aron, R. M., Weakly uniformly continuous and weakly sequentially continuous entire functions, Advances in Holomorphy, J. A. Barroso (Ed.), Math. Stud., 34, North Holland, Amsterdam (1979), 47-66.

[2] Aron, R. M. and Berner, P., A Hahn-Banach extension theorem for analytic mappings, Bull. Soc. Math. France, 106 (1978), 3-24.

[3] Aron, R. M., Boyd, C. and Choi, Y. S., Unique Hahn-Banach Theorems for spaces of homogeneous polynomials, J. Austral. Math. Soc., 70 (2001), 387-400.

[4] Aron, R. M., Hervés, C. and Valdivia, M., Weakly continuous mappings on Banach Spaces, J. Funct. Anal., 52 (1983), 189-204.

[5] Aron, R. M., Moraes, L. A. and Ryan, R., Factorization of holomorphic mappings in infinite dimensions, Math. Ann., 277(4) (1987), 617-628.

[6] Aron, R. M. and Prolla, J. B., Polynomial approximation of differentiable functions on Banach spaces, J. Reine Angew. Math., 313 (1980), 195-216.

[7] Aron, R. M. and Schottenloher, R. M., Compact holomorphic mappings on Banach spaces and approximation property, J. Funct. Anal., 21 (1976), 7-30.

[8] Davie, A. M. and Gamelin, T. W., A theorem on polynomial-star approximation, Proc. Amer. Math. Soc., 106 (1989), 351-356.

[9] Dineen, S., Holomorphy types on a Banach space, Studia Math. 34 (1977), 241-288.

[10] - Complex Analysis on Infinite Dimensional Spaces, Springer Monogr. Math., Springer Verlag, London, Berlin, Heidelberg, 1999.

[11] Floret, K., Natural norms on symmetric tensor products of normed spaces, Proc. of II Int. Workshop on Functional Analysis at Trier University, Note Mat., 17 (1997), 153-188.

[12] Gonzalez, M., Gutierrez, J. M. and Llavona, J. L., Polynomial continuity on $l_{1}$, Proc. Amer. Math. Soc., 125(5) (1997), 1349-1353.

[13] Mujica, J., Complex Analysis in Banach Spaces, Math. Stud., 120, North Holland, Amsterdam, 1986.

[14] Zalduendo, I., A canonical extension for analytic functions on Banach spaces, Trans. Amer. Math. Soc., 320 (1990), 747-763. 